Collimated prompt gamma TOF measurements with multi-slit multi-detector configurations

This content has been downloaded from IOPscience. Please scroll down to see the full text. 2015 JINST 10 P01011

(http://iopscience.iop.org/1748-0221/10/01/P01011)

View the table of contents for this issue, or go to the journal homepage for more

Download details:

IP Address: 134.58.253.57

This content was downloaded on 05/02/2016 at $16: 36$

Please note that terms and conditions apply. 


\title{
Collimated prompt gamma TOF measurements with multi-slit multi-detector configurations
}

\author{
J. Krimmer, ${ }^{a, 1}$ M. Chevallier, ${ }^{a}$ J. Constanzo, ${ }^{a}$ D. Dauvergne, ${ }^{a}$ M. De Rydt,,${ }^{a, b}$ \\ G. Dedes, ${ }^{a}$ N. Freud, ${ }^{c}$ P. Henriquet, ${ }^{a}$ C. La Tessa, ${ }^{d}$ J.M. Létang,${ }^{c}$ R. Pleskač,${ }^{d}$ \\ M. Pinto, ${ }^{a}$ C. Ray, ${ }^{a}$ V. Reithinger, ${ }^{a}$ M.H. Richard, ${ }^{a, c}$ I. Rinaldi, ${ }^{e}$ F. Roellinghoff, ${ }^{a, c}$ \\ C. Schuy, ${ }^{d}$ E. Testa ${ }^{a}$ and M. Testa ${ }^{a}$ \\ ${ }^{a}$ Institut de Physique Nucléaire de Lyon, Université de Lyon, Université de Lyon 1, CNRS/IN2P3 UMR5822, \\ 69622 Villeurbanne, France \\ ${ }^{b}$ Instituut voor Kern- en Stralingsfysica, Katholieke Universiteit Leuven, \\ Celestijnenlaan 200D, B-3001 Leuven, Belgium \\ ${ }^{c}$ Université de Lyon, CREATIS; CNRS UMR 5220; Inserm U 1044; INSA-Lyon; Université Lyon 1; \\ Centre Léon Bérard, Lyon, France \\ ${ }^{d}$ GSI Helmholtz Center for Heavy Ion Research, \\ Planckstrasse 1, D-64291 Darmstadt, Germany \\ ${ }^{e}$ Heidelberg Ion Therapy Center and Department of Radiation Oncology, \\ Im Neuenheimer Feld 450, 69120 Heidelberg, Germany \\ E-mail: j.krimmer@ipnl.in2p3.fr
}

ABSTRACT: Longitudinal prompt-gamma ray profiles have been measured with a multi-slit multidetector configuration at a $75 \mathrm{MeV} / \mathrm{u}{ }^{13} \mathrm{C}$ beam and with a PMMA target. Selections in time-offlight and energy have been applied in order to discriminate prompt-gamma rays produced in the target from background events. The ion ranges which have been extracted from each individual detector module agree amongst each other and are consistent with theoretical expectations. In a separate dedicated experiment with $200 \mathrm{MeV} / \mathrm{u}{ }^{12} \mathrm{C}$ ions the fraction of inter-detector scattering has been determined to be on the $10 \%$-level via a combination of experimental results and simulations. At the same experiment different collimator configurations have been tested and the shielding properties of tungsten and lead for prompt-gamma rays have been measured.

KEYWORDS: Instrumentation for hadron therapy; Gamma camera, SPECT, PET PET/CT, coronary CT angiography (CTA)

\footnotetext{
${ }^{1}$ Corresponding author.
} 


\section{Contents}

1 Introduction 1

2 Multi-slit experiment at GANIL 2

2.1 Experimental setup 2

2.2 Data analysis 4

$\begin{array}{lll}2.3 & \text { Results } & 6\end{array}$

3 Collimator test experiment at GSI $\quad 7$

3.1 Experimental setup

3.2 Data analysis 9

3.3 Shielding analysis 11

$\begin{array}{lll}3.4 & \text { Scattering characterization } & 13\end{array}$

$\begin{array}{ll}3.5 & \text { Influence of collimator configurations } \\ \end{array}$

4 Conclusions $\quad 16$

\section{Introduction}

The treatment of tumors via protons or carbon ions (hadrontherapy) is an emerging technique which benefits from the fact that ions deposit a large quantity of their energy close to the end of their path in the Bragg peak. This property, compared to conventional radiotherapy with X-rays, allows a better sparing of healthy tissues, in particular organs at risk close to the tumor volume. Another advantage, in the case of carbon ions, is the higher relative biological effectiveness compared to photons, due to, among others, the higher ionization density.

Due to its sharp fall-off, a major issue for quality control during treatment with ion beams is the control of the Bragg peak position and its conformation to the tumor volume. A mismatch could lead to an over-dosage in healthy tissue and an under-dosage in the target volume.

Most of the methods which are being investigated for an in vivo monitoring of the ion range during treatment are based on the detection of secondary radiation, as no primary radiation escapes from the patient. Several types of radiation have been considered for this purpose: anti-colinear $511 \mathrm{keV}$ photons following a $\beta^{+}$-decay, secondary protons from fragmentation processes (in the case of carbon ions), and prompt gammas generated at the decay of excited fragments.

The detection of $511 \mathrm{keV}$ photons [1] via positron emission tomography (PET) has already proven its applicability in hadrontherapy (see e.g. [2, 3]). However, when deducing the absorbed dose from the measured activity, biological wash-out needs to be taken into account. This is caused by long acquisition times which are required due to the limited number of produced $\beta^{+}$emitters and their relatively long decay times. 
Secondary charged particles following fragmentation processes can be detected in the case of carbon ion beams. The concept is to reconstruct the trajectories of the emitted particles and to extrapolate them to their point of creation (vertex) [4]. This interaction vertex imaging (IVI) modality is being studied by several groups [5-7].

Prompt gamma rays in the energy range up to approximately $10 \mathrm{MeV}$ are emitted nearly instantaneously after inelastic nuclear reactions of the incident ions. It has been shown that the production rate of these prompt gamma rays is highly correlated to the ion range of the primary particles [8-10], i.e. the Bragg peak position can be determined via a detection of the fall-off in the prompt gamma production rate. Systems following this approach comprise collimated cameras (both knife-edge [11, 12] and multi-slit cameras [13, 14]), as well as Compton cameras [15-20]. It has recently been shown, that with the collimated camera concept, ion ranges in homogeneous targets can be monitored with millimetric precision for a single spot in proton pencil beam scanning $[12,21]$. Time-of-flight can be used to improve the signal to background ratio, as it helps eliminating uncorrelated signals induced by neutrons and scattered particles [10, 13, 21]. This is particularly necessary for carbon ion beams. Furthermore, it is also possible to detect density variations along the ion path [22].

The collimated camera, in comparison to e.g. the Compton camera, has the advantage, that for the reconstruction no iterative algorithms need to be applied, i.e. with a multi-slit multi-detector configuration a real-time monitoring of the ion range is possible. The question of the operability of such a configuration is raised, as high energy gamma scattering between adjacent detectors could dilute the position information and therefore the information about the ion range.

The present article addresses this open question from the experimental side, with a comparison to simulations at the end. In a first experiment, measurements have been performed with a multi-slit multi-detector setup demonstrating the proof of principle. In a second step, in a separate experiment, the inter-detector scattering has been quantified. Furthermore, the influence of different collimator configurations, as well as different shieldings has been measured and results have been compared to Geant 4 simulations. These activities are an intermediate step towards a real-size prototype. The obtained data serve also as an assessment of the accuracy of the Monte Carlo simulations which are used for an optimization of the collimator dimensions.

\section{Multi-slit experiment at GANIL}

\subsection{Experimental setup}

The measurement with a multi-slit multi-detector setup was performed at GANIL (Caen, France) with $75 \mathrm{MeV} / \mathrm{u}{ }^{13} \mathrm{C}$-ions. A top-view of the experiment is given in figure 1 . Four LYSO scintillation detectors were placed at $90^{\circ}$ with respect to the beam axis behind a tungsten collimator with equidistant slits. A fifth LYSO detector was located behind a lead collimator under $90^{\circ}$ at the other side of the beam axis (see figure 1 for the dimensions). The four LYSO detectors behind the multi-slit collimator have been arranged in a way as indicated in figure 2. The LYSO crystals were coupled via lightguides to photomultiplier (PM) tubes. Symmetric and asymmetric lightguides have been combined in a way to assure a close alignment of the crystals without being hindered by the size of the PM tubes. The properties of the LYSO detectors including their crystal type and size, as well as the type of used lightguides, are denoted in table 1. 


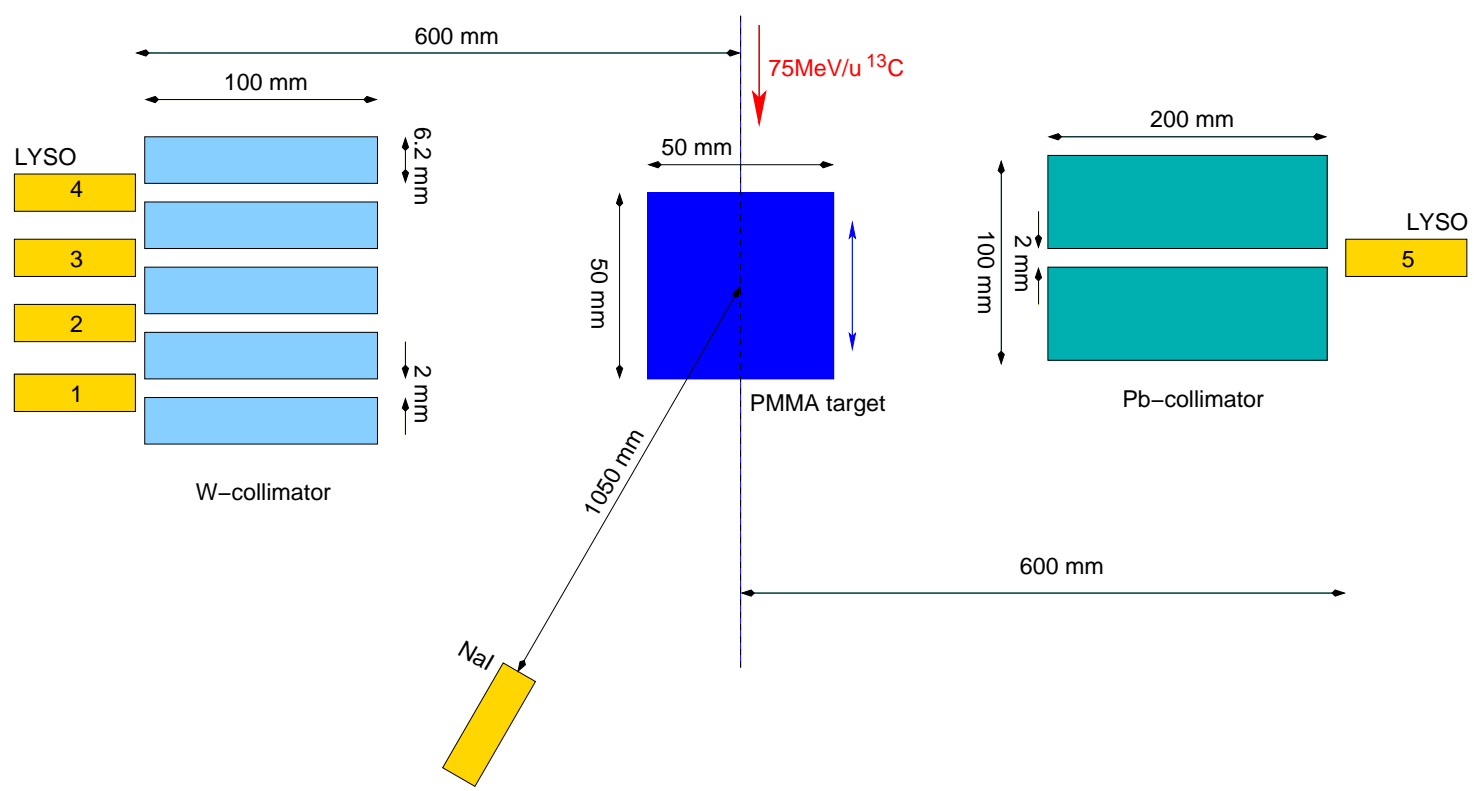

Figure 1. A multi-slit multi-detector configuration for measuring prompt gamma profiles at GANIL with $75 \mathrm{MeV} / \mathrm{u}{ }^{13} \mathrm{C}$-ions. The NaI-detector was placed underneath the target at $90^{\circ}$ with respect to the beam direction. It is represented downstream to the target for the purpose of clarity. The figure is not to scale.

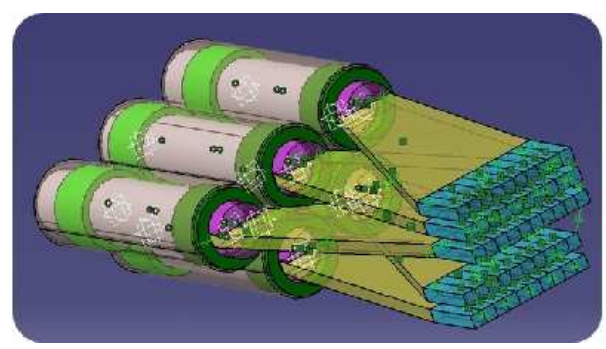

Figure 2. Indication of the arrangement of the LYSO detectors (here represented as pixellated arrays and with five detectors) behind the multi-slit collimator. A combination of symmetric and asymmetric lightguides assured a close placement of the scintillator crystals without being hindered by the size of the PM tubes.

Table 1. Properties of the used LYSO detectors, including the crystal type monolithic ('mono') or pixellated ('pixel'), the dimensions (width $\times$ height $\times$ length) and the used lightguide, asymmetric ('asy') or symmetric ('sym').

\begin{tabular}{|c|c|c|c|c|c|c|}
\hline $\mathrm{Nr}$ & 1 & 2 & 3 & 4 & 5 & 6 \\
\hline type & pixel & mono & pixel & mono & mono & mono \\
\hline size $\left[\mathrm{mm}^{3}\right]$ & $4 \times 40 \times 22$ & $5 \times 40 \times 50$ & $4 \times 40 \times 22$ & $3 \times 40 \times 50$ & $5 \times 40 \times 50$ & $3 \times 40 \times 50$ \\
\hline lightguide & sym & asy & asy & asy & sym & sym \\
\hline
\end{tabular}


A cubic polymethyl-methacrylate (PMMA) target $\left(50 \times 50 \times 50 \mathrm{~mm}^{3}\right)$ was mounted on a translation table. Longitudinal prompt-gamma ray profiles have been obtained by a scan of the target in front of the detectors. The detector signals were coupled to standard NIM electronics and data were registered via a VME data acquisition system. For every detector an ADC information is available, whereas only one time information from a TAC (time-to-amplitude converter) was provided. The start signal for the TAC came from the logic-OR of the five LYSO detectors after passing constant fraction discriminators, whereas the high frequency (HF) signal of the accelerator (one bunch of $1 \mathrm{~ns}$ every $80 \mathrm{~ns}$ ) served as stop. For a normalization to the incident ion flux, a NaI detector was installed at $1050 \mathrm{~mm}$ from the target for the registration of secondary radiation from the primary beam. This detector was placed underneath the target table at $90^{\circ}$ with respect to the beam direction. The count rate of the $\mathrm{NaI}$ detector was checked to be independent of the target position. It has been calibrated via a Faraday cup. Energy calibration of all detector modules has been performed with radioactive sources.

\subsection{Data analysis}

For the five LYSO detectors only a single TAC had been available to give the time-of-flight (TOF) information between a start produced by an event in a detector module and the stop from the (delayed) accelerator HF. This time information needs then to be assigned to the individual detectors in the offline analysis. For each module a software threshold $(300-400 \mathrm{keV})$ is defined which is equal to the associated hardware discriminator threshold. In order to avoid ambiguities in the assignment of the TAC information, only events with multiplicity 1 have been taken into account in a first analysis step. With this restriction only one module has an ADC entry above its software threshold and the entries of all other modules are below their respective thresholds. Figure 3 shows the energy versus TOF spectra under these conditions for the five LYSO detectors. The spectra reveal a horizontal band below $1 \mathrm{MeV}$ which can be attributed to the internal radioactivity of the LYSO detectors ( $\beta^{-}$-decay of ${ }^{176} \mathrm{Lu}$ to the $597 \mathrm{keV}$ excited state of ${ }^{176} \mathrm{Hf}$ followed by a cascade of $3 \gamma$-rays [23]) and to $511 \mathrm{keV}$ lines. The (close-to) vertical bands at $t \approx 25 \mathrm{~ns}$ contain the promptgamma rays. This part is mostly pronounced for LYSO 3 and LYSO 5 as for the actual target position the Bragg-peak is close to the field of view of these two detector modules. At this particular position, the beam path in the PMMA block is not in the field of view of LYSO 1 and only marginally for LYSO 2. The absolute time position of the prompt-gamma band depends on cable lengths and individual photomultiplier transit times only and is not relevant for the further analysis.

Besides the prompt gammas, also neutrons or neutron induced secondary gamma rays are registered. These events can be identified via their larger TOF values (with respect to the promptgamma rays). As neutrons can traverse the collimator, no correlation with the incident ion range can be observed [10]. Therefore, these events contribute to background, only. For a selection of the relevant events, energy and TOF selections as described in [10, 13], are applied.

The red curve in figure 4 is the TOF spectrum of LYSO 3 for a target position which is close to the Bragg peak for this detector (note that the target can be moved in front of the detectors (figure 1)). A timing window (here with a width of $3.6 \mathrm{~ns}$ ) is selected around the prompt-gamma peak. The widths of the prompt-gamma windows have been adapted for the individual LYSOs and their positions respect the change of TOF at different target positions. 

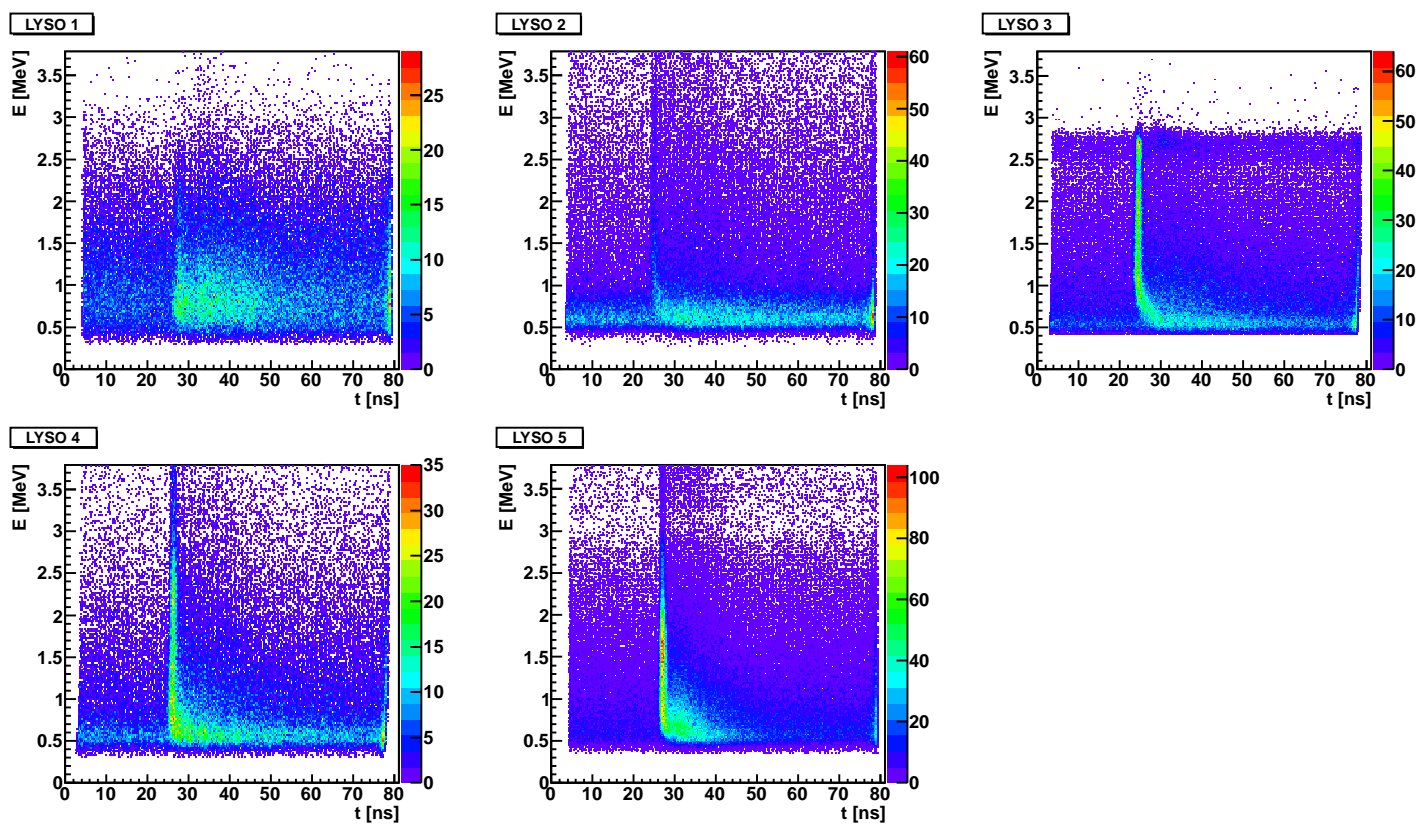

Figure 3. Energy versus TOF spectra for the five LYSO detectors under the condition of multiplicity 1. The origin of time (horizontal axis) is arbitrary. The z-axis (color-scale) represents the number of counts per bin ( $\Delta t=0.3 \mathrm{~ns}, \Delta E$ between 15 and $22 \mathrm{keV}$ depending on the energy calibration). The (close-to) vertical bands around $25 \mathrm{~ns}$ correspond to prompt-gamma rays. Neutrons or neutron induced gamma rays with their larger TOF values show up on the right of the prompt-gamma bands. ${ }^{2}$

In order to account for background in these prompt-gamma regions, reference data have been taken at two positions, one before the target entrance, and the other after the Bragg peak. The blue curve in figure 4 represents the mean spectrum of these two measurements after applying a scaling factor. This factor is the ratio of integrals outside the prompt region for the TOF spectrum in the Bragg peak position and the reference data. The integrals in these regions increase almost linearly along the beam axis as neutron emission is not isotropic, but favors forward directions [10, 24]. By using reference data before the target and after the Bragg peak and the application of a scaling factor, this effect is taken into account (see also [22]). Finally, the events remaining in the prompt region, after subtracting the background, are integrated. Information from the calibrated $\mathrm{NaI}$ detector is used for a normalization to the number of incident ions.

A further selection of relevant events in the data sample is performed via cuts in the energy regime. Above $1 \mathrm{MeV}$ the events attributed to the internal radioactivity of LYSO are sufficiently suppressed. With the present setup the number of events for which a photon has undergone scattering in a neighboring detector module could not be quantified due to the use of a single TAC only. The signature from the deposited energy (ADC information) is not sufficient for this purpose, as these signals comprise also random events which are acquired during the ADC gate of 4-5 $\mu$ s (at GANIL every $80 \mathrm{~ns}$ a bunch of ions arrives). A dedicated experiment has therefore been performed at GSI, which will be described in section 3 .

\footnotetext{
${ }^{2}$ Low energy neutrons from a previous bunch can also appear left to the prompt-gamma band.
} 


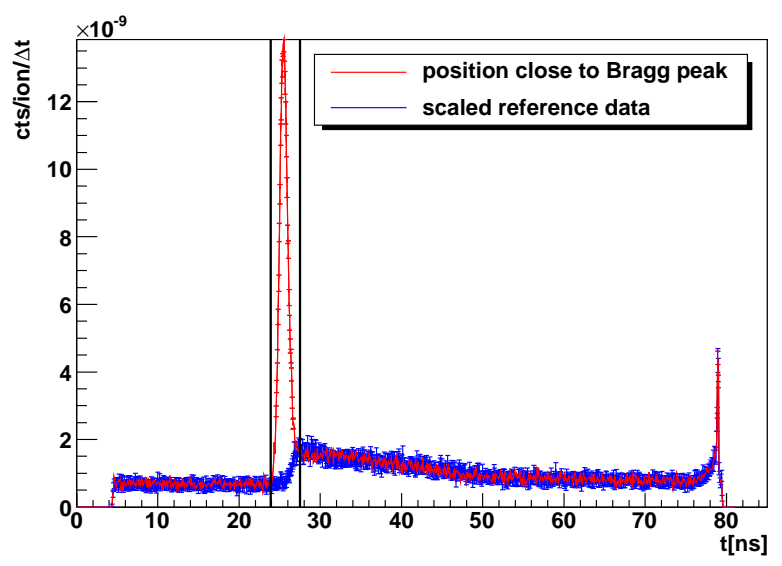

Figure 4. TOF spectra for LYSO 3 for a position close to the Bragg peak (in red) and a scaled mean spectrum (in blue) from two reference points, one before the target, and one after the Bragg peak. The vertical lines indicate the prompt region. These spectra contain only events with energies above $1 \mathrm{MeV}$. The ordinate represents the number of counts per incident ion and time bin $\Delta t=0.8 \mathrm{~ns}$.

\subsection{Results}

With the multi-slit multi-detector configuration data have been taken at 24 different target positions in order to obtain longitudinal prompt-gamma profiles (GANIL $75 \mathrm{MeV} / \mathrm{u}$ ions). In figure 5 a) the registered counts per incident ion are plotted as a function of the target position. For this figure an energy threshold of $400 \mathrm{keV}$, but no TOF selection, has been applied. In this plot the structures which are due to the target entrance and the range of the incident ions are dominated by background. In figure $5 \mathrm{~b}$ ), a $400 \mathrm{keV}$ energy threshold has been applied and TOF information has been included. Furthermore, background has been subtracted, as described in the previous section. As only for LYSO 2 reference points with sufficient distance to the target entrance and to the falloff exist, a single reference point has been used for the background subtraction of the other detector modules. Namely the point at $+14 \mathrm{~mm}$ for LYSO 1 and $-30 \mathrm{~mm}$ for LYSO 3-5, respectively.

a)

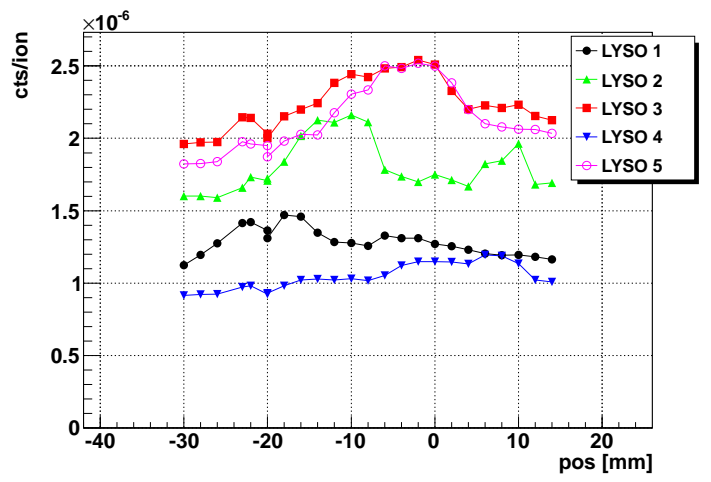

b)

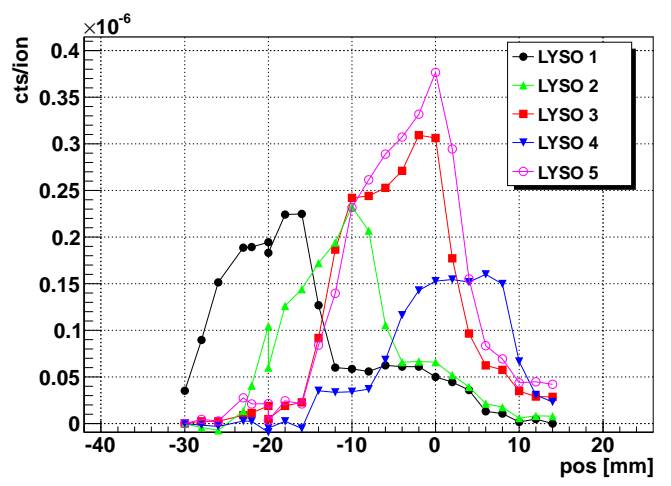

Figure 5. Longitudinal prompt-gamma ray profiles. a) With a 400-keV energy threshold, but without TOF selection. b) With a 400-keV energy threshold, including TOF information and after background subtraction. 
a)

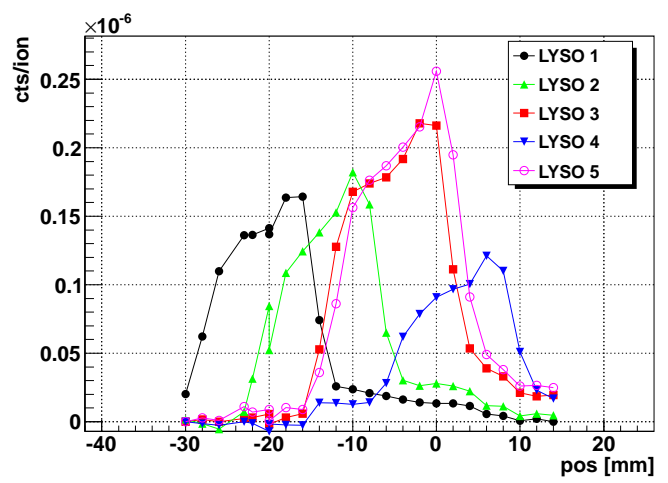

b)

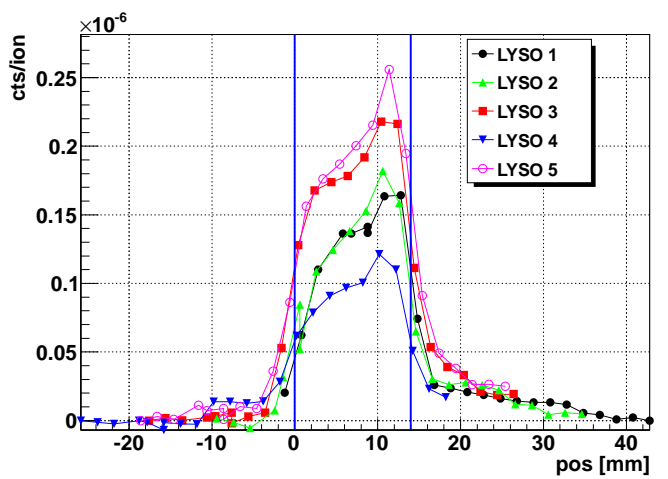

Figure 6. a) Longitudinal prompt-gamma ray profiles with TOF selection, a 1-MeV energy threshold and under the condition of multiplicity 1 . b) The detector positions have been taken into account and the horizontal axes have been shifted accordingly, for a better comparison of the results. The vertical blue lines represent the target entrance and the range of ${ }^{13} \mathrm{C}$ ions, respectively.

In figure $5 \mathrm{~b}$ ) the increase of count rate at the target entrance and the fall-off in the Bragg-peak region can be identified. For LYSO 1 and LYSO 2 another bump after the Bragg peak, around $0 \mathrm{~mm}$, appears. A possible origin of these events might be Compton scattering from neighboring detector modules. Another contribution comes from random events which are acquired during the ADC gate of 4-5 $\mu \mathrm{s}$. For a correct identification and quantification of scattered events via the timing signature, every detector module needs to be equipped with a TDC, however, which was not the case for the present experiment. We refer to sections 3.2 and 3.4.

With an increase of the threshold to $1 \mathrm{MeV}$ and a restriction to multiplicity 1 , the bump around $0 \mathrm{~mm}$ can be minimized. ${ }^{3}$ The results under these conditions are shown in figure $6 \mathrm{a}$ ).

For a better comparison of the profiles from the individual detectors, the detector positions have been taken into account and the horizontal axes have been shifted accordingly (figure 6 b)). The vertical blue lines represent the positions of the target entrance and the range of $75 \mathrm{MeV} / \mathrm{u}{ }^{13} \mathrm{C}$ ions in PMMA, respectively. Although the count rates are not the same due to different detector geometries (see table 1), the target entrance and the fall-off is revealed at positions which are consistent with the theoretical values. For a quantitative investigation of the range retrieval precision as a function of the available statistics in the case of incident protons see ref. [21].

\section{Collimator test experiment at GSI}

\subsection{Experimental setup}

For an investigation of the scattering between detectors, as well as the influence of different collimator configurations and shieldings, a dedicated experiment has been performed with $200 \mathrm{MeV} / \mathrm{u}$ ${ }^{12} \mathrm{C}$-ions ${ }^{4}$ at GSI (Darmstadt, Germany). A top-view of the complete setup is displayed in figure 7.

\footnotetext{
${ }^{3}$ For a complete suppression of scattered events the TOF information of the individual detector modules would be necessary.

${ }^{4}$ The shape of the prompt-gamma energy spectrum in the range $1-10 \mathrm{MeV}$ shows only minor influence from the primary ion energy. For the proton case see e.g. [25, 26].
} 
The carbon ions passed a beam tagging hodoscope, consisting of an array of orthogonal scintillating fibers, which provided a time and position information. As target served a 200-mm long PMMA cylinder with a diameter of $150 \mathrm{~mm}$. Produced secondary radiation was registered with detectors placed under $90^{\circ}$ with respect to the incident beam direction. LYSO detectors behind a collimator were used for the investigation of inter-detector scattering, as well as for the test of different collimator configurations. Shielding properties were measured with a cylindrical $\mathrm{LaBr}_{3}$ detector (diameter: $25.4 \mathrm{~mm}$, length: $50.8 \mathrm{~mm}$ ) mounted behind lead or tungsten blocks, with various thicknesses.

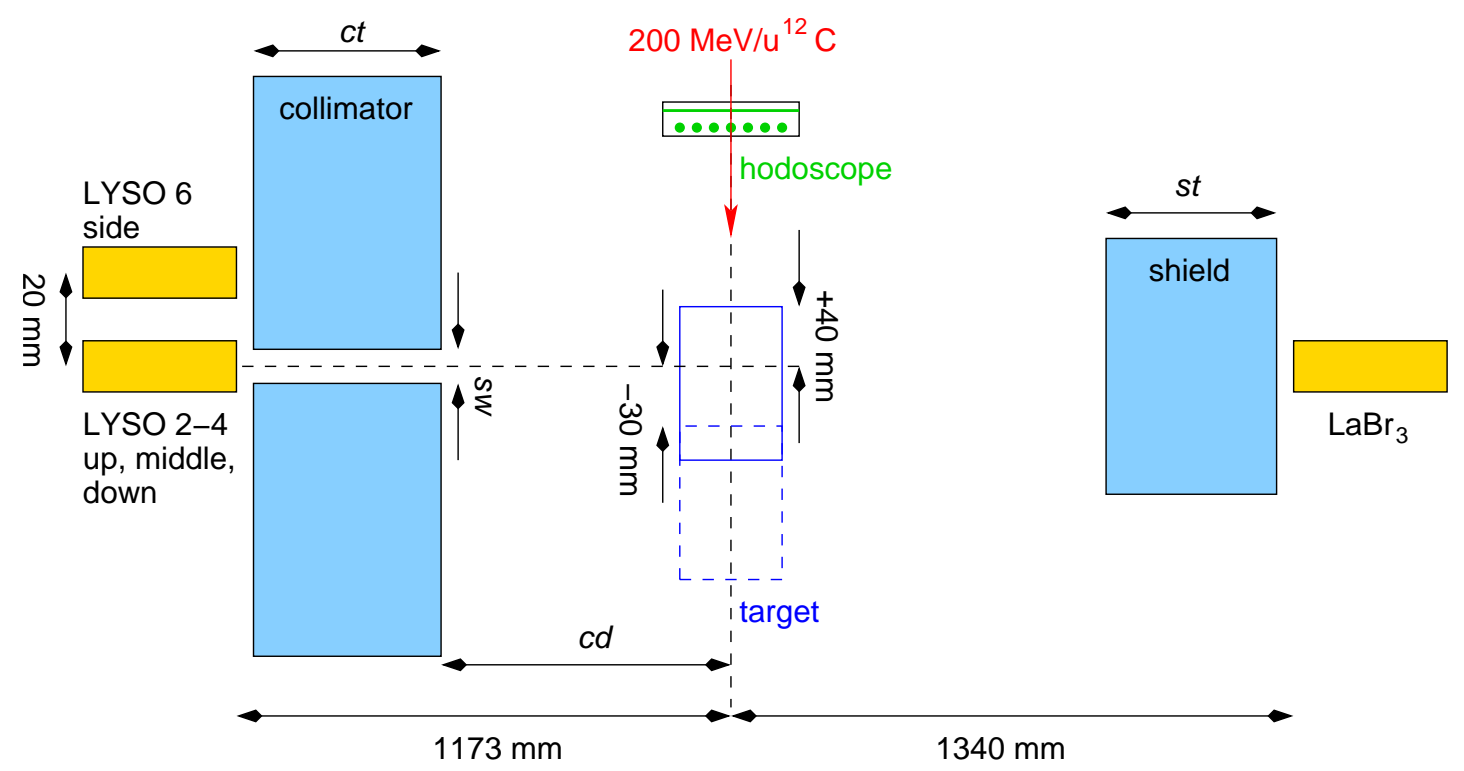

Figure 7. Setup for the investigation of inter-detector scattering and the influence of different collimator configurations and shieldings with $200 \mathrm{MeV} / \mathrm{u}{ }^{12} \mathrm{C}$-ions at GSI. The figure is not to scale.

LYSO 2-4 (following the labeling of table 1) were placed behind the slit of the collimator, whereas LYSO 6 had no direct view to the target (see figure 8 for a side-view of the detector arrangement behind the collimator). With exception of LYSO 6 the used detector modules were the same as for the previously described experiment at GANIL. The dimensions are given in table 1.

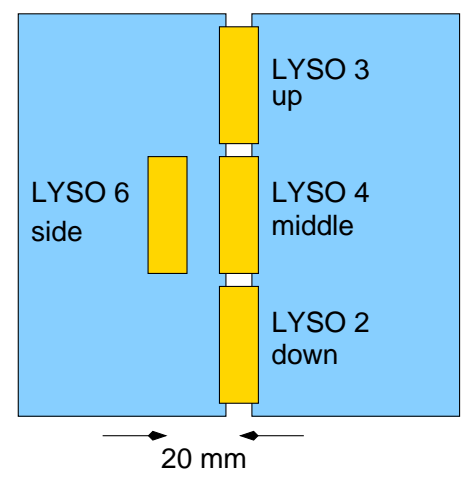

Figure 8. Arrangement of the LYSO detectors behind the collimator for the inter-detector scattering experiment. 
The detector signals were connected to standard NIM electronics, and data were recorded with a VME data acquisition system. For each detector an ADC and TDC information is available. The delayed signal from the beam tagging hodoscope served as time reference for the TDC (stop signal).

Different configurations have been tested, where the collimator material, the collimator distance $c d$, the slit width $s w$ and the collimator thickness $c t$ have been varied, whereas the distance between the target and the LYSO detectors was kept fixed at $1173 \mathrm{~mm}$. Two major configurations have been tested, one with the collimator 'far' from the target, next to the detectors, and another with the collimator more 'close' to the target. An overview of the parameters for the different collimator configurations is given in table 2. The test with different shielding materials ( $\mathrm{W}$ and $\mathrm{Pb}$ ) and thicknesses st (between 0 and $200 \mathrm{~mm}$ ) was performed at a fixed distance $(1340 \mathrm{~mm}$ ) between the target and the $\mathrm{LaBr}_{3}$ detector.

Table 2. Different collimator configurations comprising a variation of the material, the collimator distance $c d$, the slit width $s w$, and the collimator thickness $c t$.

\begin{tabular}{|c|c|c|c|c|}
\hline configuration & material & distance $(c d[\mathrm{~mm}])$ & $s w[\mathrm{~mm}]$ & $c t[\mathrm{~mm}]$ \\
\hline 0 & $\mathrm{~Pb}$ & far $(1073)$ & 4 & 100 \\
\hline 1 & $\mathrm{~Pb}$ & far $(1123)$ & 4 & 50 \\
\hline 2 & $\mathrm{~W}$ & far $(1073)$ & 4 & 100 \\
\hline 3 & $\mathrm{~W}$ & far $(1123)$ & 4 & 50 \\
\hline 4 & $\mathrm{~Pb}$ & close $(760)$ & 4 & 100 \\
\hline 5 & $\mathrm{~Pb}$ & close $(760)$ & 8 & 100 \\
\hline
\end{tabular}

\subsection{Data analysis}

At this experiment each detector element has been equipped with a TDC in order to determine the number of events which have been scattered from another detector module, via their timing signature.

In a first analysis step, energy versus TOF spectra of LYSO 2-4, the three detectors behind the slit of the collimator, have been produced (figure 9). The vertical band is due to prompt gamma rays which are generated in the target and are passing the slit of the collimator. The vertical structures which appear in the upper and middle LYSO more than $20 \mathrm{~ns}$ before the prompt band can be attributed to beam diagnostic elements upstream to the target area. For a selection of the relevant events, cuts in the energy and time domain have been defined as indicated by the red lines in the figure.

As LYSO 6 has no direct view to the target, it is only sensitive to scattered events from LYSO 24 or to background. Scattered events can be identified via their time signature. As they are synchronous with an event in LYSO 2-4 the time difference results in a constant value which is only determined by cable lengths and transition times of the electronics modules. For background events in LYSO 6 there would be no such time correlation with the other detector modules. The results of the time differences $t_{\text {diff }}$ of the detector at the side (LYSO 6) to the three other detectors (up, middle, down) are given in figure 10. For these time distributions prompt-gamma rays have been 
selected, i.e. for LYSO 2-4 only events inside the red regions of figure 9 have been considered. Furthermore, the same energy window has also been selected for LYSO 6. It should be noted that for this study only configurations with a collimator length of $100 \mathrm{~mm}$ have been used in order to minimize the amount of photons which can penetrate the collimator, as these photons would falsify the measured number of scattered events (see also section 3.4). The statistics in figure 10 for the upper and lower detector is very limited whereas the time difference of the middle detector reveals the signature of scattered events with its concentration around a constant value. The number of entries in these spectra can therefore directly be attributed to scattered prompt-gamma events with origin LYSO 2-4.
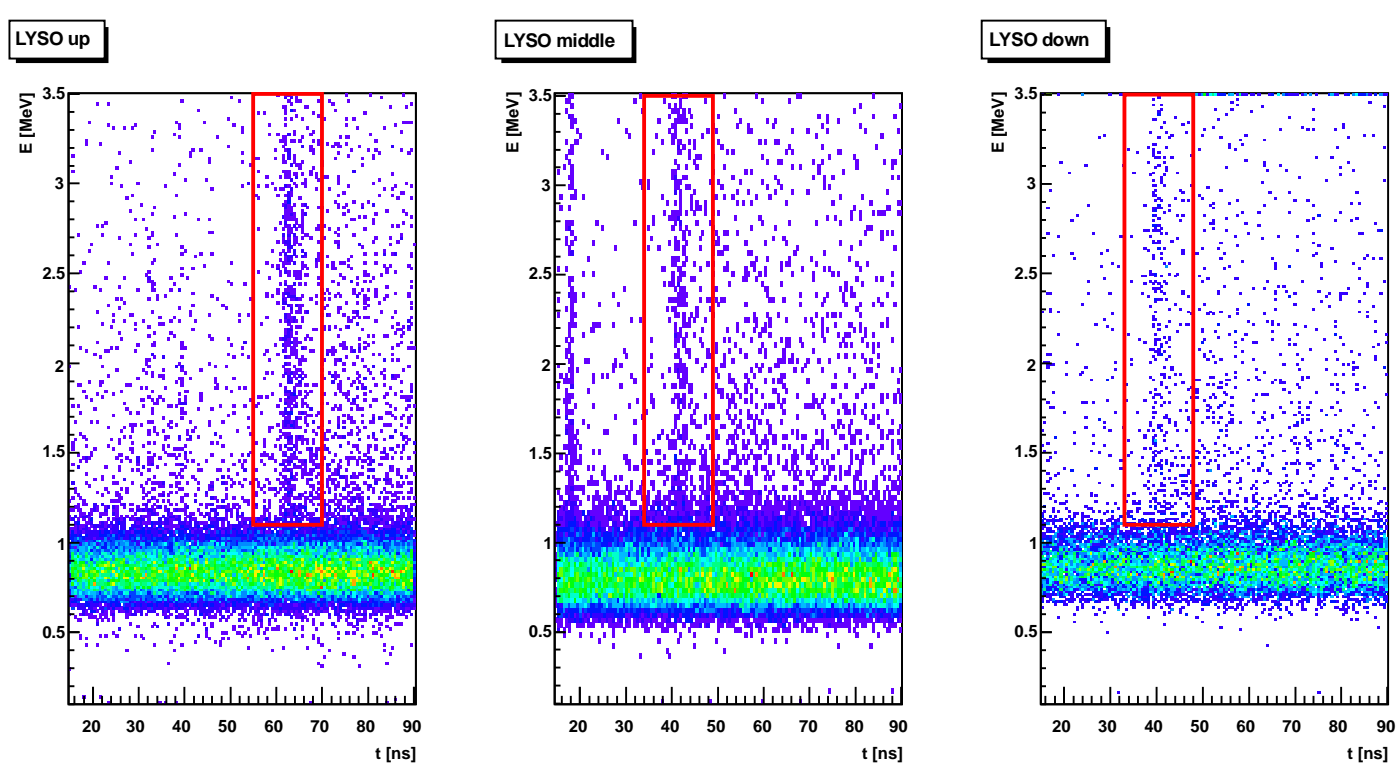

Figure 9. Energy versus TOF spectra for the three LYSO detectors behind the collimator slit. The red lines indicate the regions of selected prompt gamma ray events. The origin of the time scale is arbitrary.
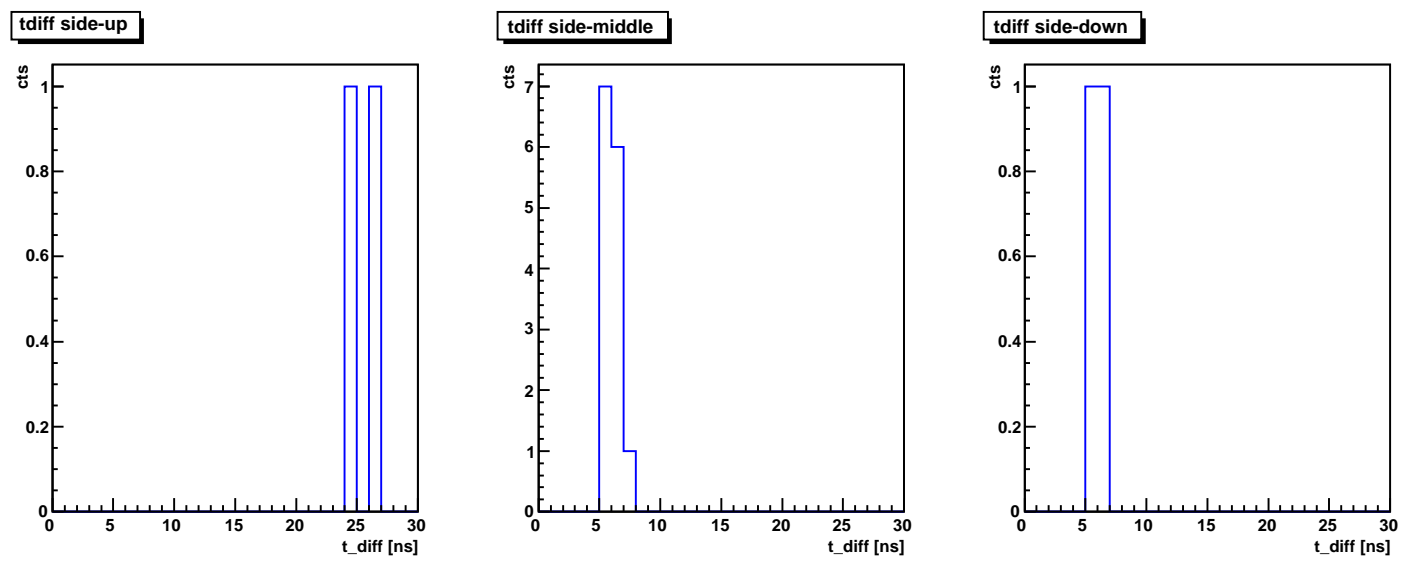

Figure 10. Time differences of the detector at the side (LYSO 6) to the three other detectors (up, middle, down). 
With the same experimental setup the influence of different collimator configurations to the contrast in prompt gamma ray profiles has been investigated. For the previously described experiment at the cyclotron of GANIL the accelerator HF could be used as time reference for the TOF measurements. As this is not possible for the synchrotron of GSI, a detector intercepting the beam needed to be used (figure 7), which limited the rate of incident ions to less than $10^{6} 1 / \mathrm{s}$. Under these conditions the measuring time for a single data point is on the order of 15 minutes. The total available beamtime for this type of studies was 5 hours which led to the restriction to take data for each configuration at two target positions only. One at $-30 \mathrm{~mm}$ before the target and the other at $+40 \mathrm{~mm}$ inside the target (see figure 7). The corresponding TOF spectra of the three detectors behind the collimator slit (configuration 0 , see table 2) are given in figure 11, where the two target positions are represented by the blue and red lines, respectively. The number of events are obtained via integration in the prompt-gamma region indicated by the vertical lines in the figure. At the configuration 0 (lead collimator with a thickness of $100 \mathrm{~mm}$ ) the blue spectra reveal also a prompt-gamma peak, i.e. prompt gamma rays are not completely absorbed by the collimator material.
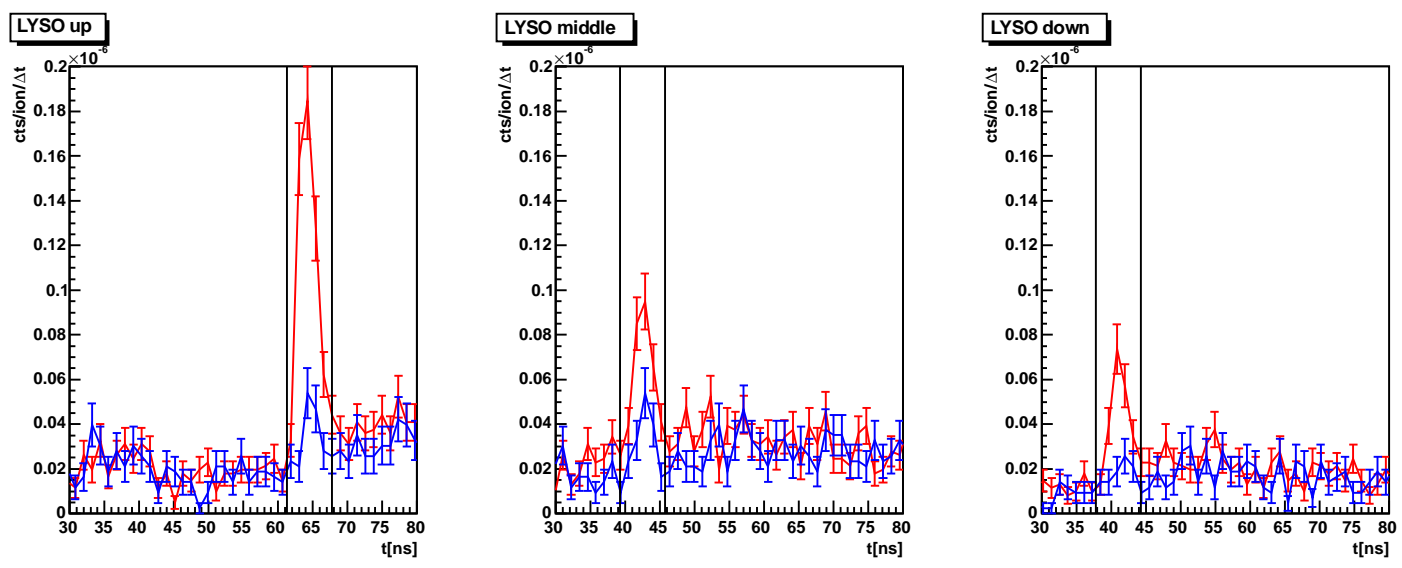

Figure 11. TOF spectra of the three LYSO detectors behind the collimator slit for configuration 0 (table 2). The red (blue) lines represent a point with a field of view of the ion range inside (before) the target. The ordinate displays the counts per incident ion and time bin $\Delta t=1.2 \mathrm{~ns}$

For an investigation of shielding properties and transmission of prompt gamma rays by different materials, the $\mathrm{LaBr}_{3}$ detector has been placed behind lead or tungsten layers with various thicknesses, respectively (see figure 7). Figure 12 shows the TOF spectra obtained from the $\mathrm{LaBr}_{3}$ without shielding (red line), with $50 \mathrm{~mm}$ (blue line) and $100 \mathrm{~mm}$ (black line) thick lead bricks in front of the detector, as well as with a $75 \mathrm{~mm}$ tungsten shielding (green line). Furthermore, results from Geant4-simulations [27] (version 9.6.p02) are also given for the configuration with $100 \mathrm{~mm}$ lead shielding (purple line). For all the simulations in this article the reference physics list (QGSP_BIC_HP), which involves the binary light ion cascade model, has been applied.

\subsection{Shielding analysis}

As a measure of the prompt gamma rays which can pass through the shielding, count rates of the $\mathrm{LaBr}_{3}$ detector have been integrated in time windows around the prompt gamma peak (figure 12). 


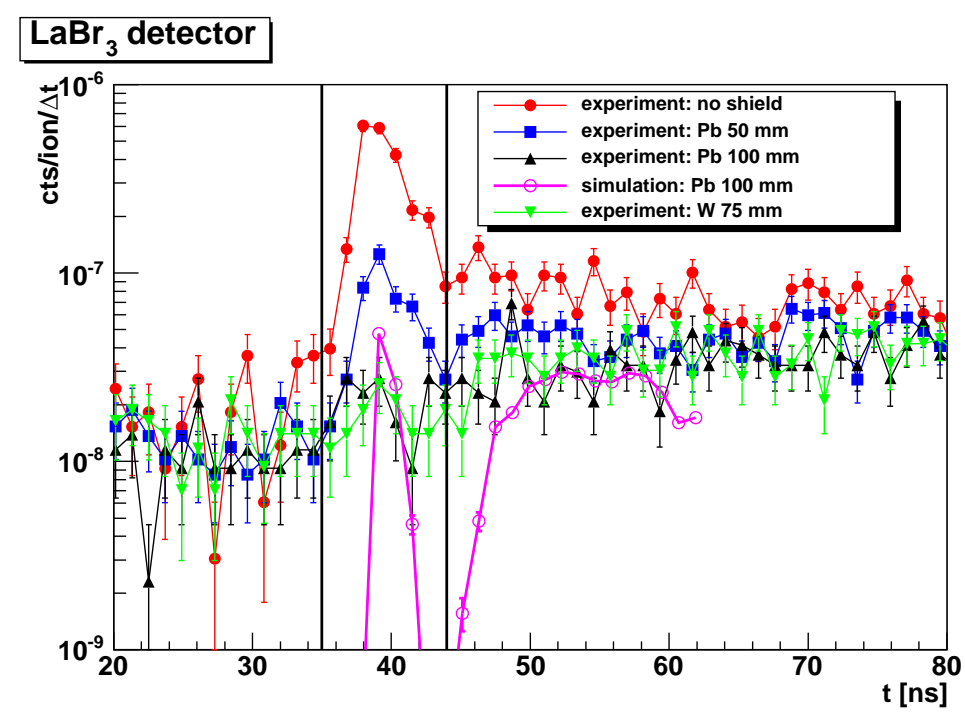

Figure 12. Experimental TOF spectra from the $\mathrm{LaBr}_{3}$ detector without shielding (red line), with $50 \mathrm{~mm}$ and $100 \mathrm{~mm}$ thick lead layers (blue and black lines), and with a $75 \mathrm{~mm}$ thick tungsten shielding (green line). For comparison simulated data is also shown for the $100 \mathrm{~mm}$ lead shielding (purple line). The ordinate represents the counts per incident ion and time bin $\Delta t=1.2 \mathrm{~ns}$.

The required deposited energy was between 1.1 and $10 \mathrm{MeV}$. The results for different shielding materials and thicknesses are given in figure 13.

For the points without shielding (thickness 0 ) the simulated prompt-gamma production overshoots the measured one. This effect has already been reported in the literature [28]. The discrepancy can be reduced via the use of the Quantum Molecular Dynamics (QMD) model and a tuning of the free parameters therein [29]. As for the present comparison with experiment the absolute prompt-gamma rate is not of utmost importance, and for the sake of comparability, this approach has not been followed, and the reference physics list (QGSP_BIC_HP) has been applied, instead.

Simulated data reveal a linear behavior in the semi-logarithmic plot of figure 13 over the whole range for $\mathrm{Pb}$ and $\mathrm{W}$. The experimental data with the lead shielding indicate saturation effects already at a shielding thickness of $100 \mathrm{~mm}$, whereas the simulated data continue to decrease with increasing shielding. This can be clarified via the TOF spectra of figure 12. In the experimental data with a $100 \mathrm{~mm}$ lead shielding no prompt-gamma peak is revealed, i.e. the integral is dominated by other events, e.g. neutrons or neutron-induced gammas which might have also been produced in a previous bunch. In the simulation, however, the integral is dominated by the prompt-gamma peak, especially no entries before the prompt-gamma peak appear. This is due to the idealization in the simulation which does not take into account the time structure of the beam, which means that no events from previous ion bunches appear. Furthermore, the experimental hall has not been modeled which leads to a reduction of background in the simulated spectra. Finally, a perfect timing resolution of the $\mathrm{LaBr}_{3}$ detector has been assumed.

Regarding the shielding properties of lead and tungsten, the latter is more efficient due to its density which is a factor 1.6 larger compared to lead. This difference in the shielding properties is more clearly revealed in the simulations than in the experimental data, as for the measured point 


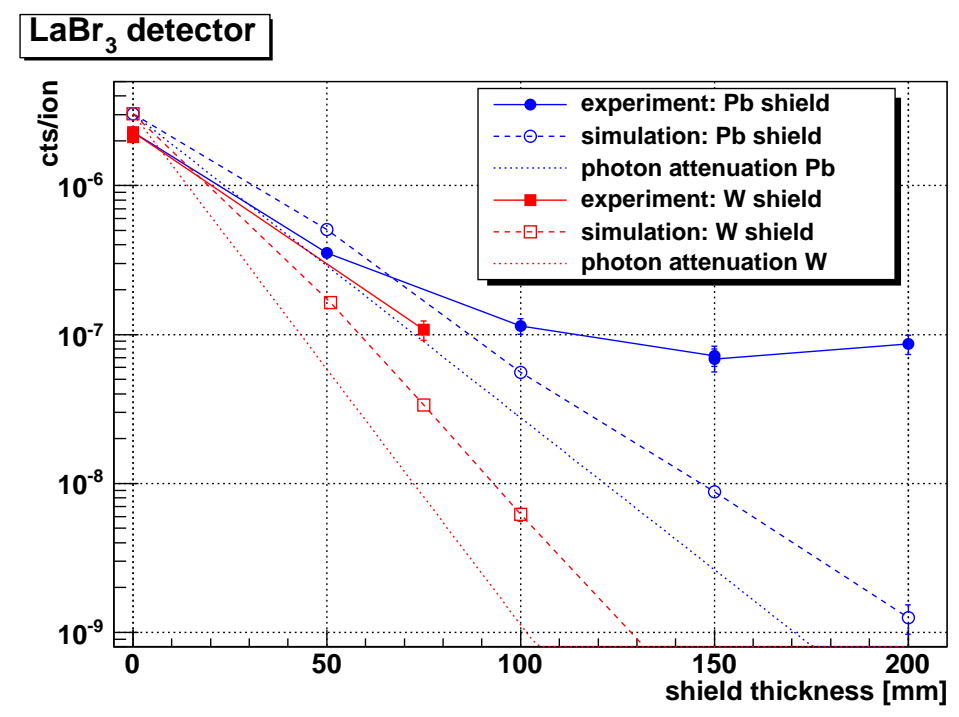

Figure 13. Integrated count rates of the $\mathrm{LaBr}_{3}$ detector behind lead and tungsten shielding with various thicknesses in comparison with Geant4 simulations and the attenuation of photons impinging the two shielding materials.

with a $75 \mathrm{~mm}$ tungsten shielding the integral in the TOF spectrum is already dominated by other events than prompt-gamma rays (figure 12).

For comparison figure 13 shows also the exponential attenuation of the photon flux according to the Lambert-Beer law. The initial count rates have been set to the ones obtained via simulations. The mass attenuation coefficients of $0.041 \mathrm{~cm}^{2} / \mathrm{g}$ for $\mathrm{W}$ and $0.042 \mathrm{~cm}^{2} / \mathrm{g}$ for $\mathrm{Pb}$ have been taken from [30] at $3 \mathrm{MeV}$ which represents a medium energy in the prompt-gamma spectrum. Note that this energy corresponds to the minimum value of the attenuation coefficients.

Also here the different slopes for $\mathrm{Pb}$ and $\mathrm{W}$ indicate that the latter is more efficient for shielding due to its larger density. In this idealized exponential case the attenuation is more pronounced than for the simulations or the experimental data. This is caused by the fact that in the Lambert-Beer law every interaction leads to a reduction of the initial flux, whereas in the simulation or experimental case photons which have undergone an interaction in the shielding, e.g. a Compton scattering, could still reach the (finite-size) detector.

\subsection{Scattering characterization}

In a multi-slit multi-detector setup with the intended purpose of measuring longitudinal promptgamma profiles, inter-detector scattering of prompt-gamma rays would lead to a dilution of position information, as for the reconstruction of the profiles it is assumed that the photons passed the corresponding collimator slit in front of the detector. For scattered events this assumption is not valid anymore. The amount of this inter-detector scattering has been determined at a dedicated experiment at GSI with three LYSO detectors behind the collimator slit in coincidence with one detector at the side, without a direct view to the target (see figure 8). The results are summarized in table 3. The number of scattered prompt-gamma ray events which have been registered with LYSO 6 were extracted from figure 10. The total number of prompt-gamma rays in LYSO 2-4 
Table 3. Scattered and total number of prompt-gamma ray events for the three LYSO detectors behind the collimator slit. The number of scattered events (in coincidence with LYSO 6 positioned as in figure 8) were extracted from figure 10, whereas the total numbers were accumulated inside the regions indicated by the red lines of figure 9.

\begin{tabular}{|c|c|c|c|}
\hline & LYSO 3 (up) & LYSO 4 (middle) & LYSO 2 (down) \\
\hline scattered & 2 & 14 & 2 \\
\hline total & 1098 & 791 & 481 \\
\hline ratio & $(0.2 \pm 0.1) \%$ & $(1.8 \pm 0.5) \%$ & $(0.4 \pm 0.3) \%$ \\
\hline ratio (Geant4) & $(0.5 \pm 0.1) \%$ & $(2.2 \pm 0.2) \%$ & $(0.2 \pm 0.1) \%$ \\
\hline
\end{tabular}

were accumulated inside the regions indicated by the red lines of figure 9. Most of the scattered events have their origin in the middle detector (LYSO 4) which is next to LYSO 6 (figure 8). The simulations have been performed with the geometry of configuration $0(100 \mathrm{~mm} \mathrm{~Pb}$ collimator, table 2) and the same time and energy windows have been applied as for the experimental data analysis. The results are in good agreement with the experimental data.

The above described method for determining the number of scattered events relies on the hypothesis that LYSO 6 detects only scattered events, i.e. photons which have originally passed the slit of the collimator and which have then diffused in one of the detectors behind the slit. Photons traversing the bulk part of the collimator and having an interaction in LYSO 6 could also be scattered to one of the other detectors. Experimentally this type of events cannot be discriminated by the first one and would lead to a falsification of the measured number of scattered events. For a minimization of penetrating photons, only collimator configurations with a length of $100 \mathrm{~mm}$ have been analyzed, therefore. Here, according to the simulations (cf. figure 13) only $2 \%$ of the initial photons traverse a $100 \mathrm{~mm}$ thick $\mathrm{Pb}$ collimator $(0.2 \%$ for $100 \mathrm{~mm} \mathrm{~W})$. In the experiment this part is $6 \%$ for $\mathrm{Pb}$ and less than $5 \%$ for $\mathrm{W}$. From this it follows that the measured or simulated number of scattered events might have been overestimated by $6 \%$ or $2 \%$ (relative uncertainty), respectively. This uncertainty is well within the statistical error stated in table 3.

For a further investigation of the inter-detector scattering the measured configuration from figure 8 has been extended to a simulated set-up with three rows of LYSO detectors (figure 14 left). The dimensions and position of the collimator correspond to configuration 0 from table 2 . The detectors in the bottom and top row have the same dimensions as LYSO 2 and LYSO 3, respectively. The detectors in the middle row (L6_1-L6_5) are further investigated as they can detect scattered events from their horizontal neighbors, as well as diagonal from above and below. Vertical scattering has been ignored as it does not lead to a degradation of the position information. Scattered events are defined as prompt-gammas having an interaction in one of the detectors behind the slit (LYSO 2-LYSO 4) and a synchronous hit (within $1 \mathrm{~ns}$ ) in one of the detectors behind the bulk part of the collimator. An energy window from 1.1 to $10 \mathrm{MeV}$ has been set for all detectors. The right part of figure 14 gives the ratio of scattered events detected in the additional detectors (L6_1-L6_5) to the number of events in their origin detector (LYSO 2-LYSO 4). The points at zero correspond to LYSO 6. Horizontal scattering (here the part with origin LYSO 4) is the dominating part for the next neighbor and then decreases rapidly to negligible values. Diagonal scattering from the detectors above and below (here LYSO 2 and LYSO 3) plays also a role in its totality. 

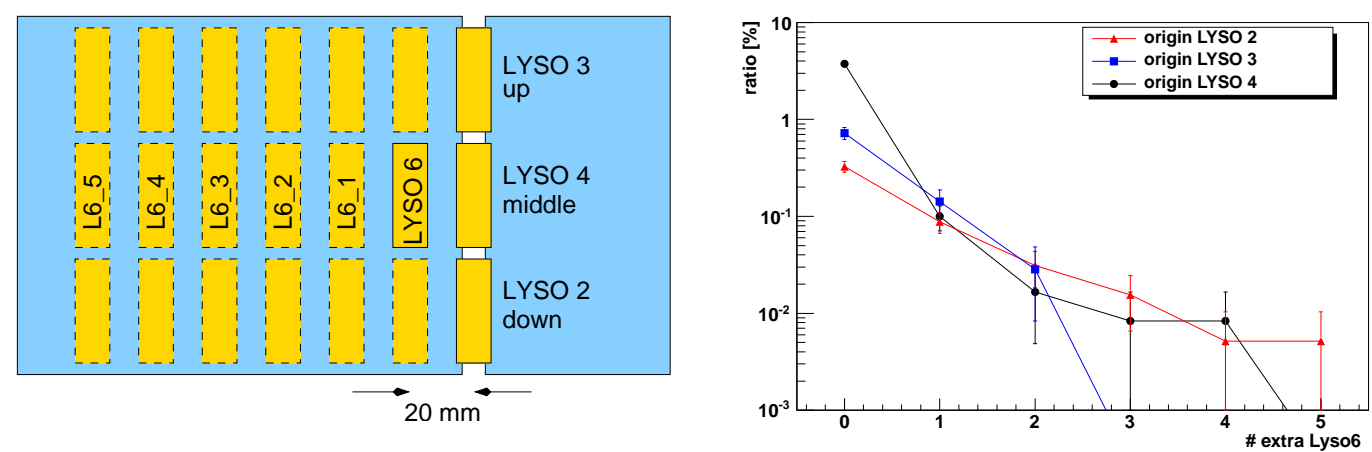

Figure 14. Left: simulated configuration with three rows of LYSO detectors. The five additional LYSO detectors (L6_1-L6_5) in the middle row are used for a further investigation of inter-detector scattering. Right: results from the simulation. Ratio of detected events in the extra LYSO detectors to the number of events in their origin detector.

For an estimation of the average portion of scattering, the middle detector behind the slit (LYSO 4) has been selected as reference. The hypothesis is, that the fraction of scattered events from this detector to the other detector modules is the same as the fraction of scattered events from all other detector modules, arriving in LYSO 4. This module has been selected as reference, as from here horizontal, as well as, diagonal scattering can occur. The results will therefore state an upper limit of the scattering portion. The total fraction of events with an interaction in LYSO 4 and a synchronous hit in one of the other detector modules amounts to $(4.8 \pm 0.2) \%$. The here described simulated results contain only diffusion to one direction (here from LYSO 4 to the left). In a realistic scenario for a detector in the middle of a row, scattering can occur to both directions, i.e. the obtained ratio needs to be multiplied by two, resulting in $(9.6 \pm 0.4) \%$. The above described penetration of the bulk part of the collimator by photons would lead to a $2 \%$ relative diminution of this value, i.e. $10 \%$ diffusion can be stated as a ballpark figure for the scattering fraction. This rough estimate has been extracted under the assumption of equal count rates for neighboring detectors which is certainly valid in the plateau region of the Bragg curve. Deviations will be expected in the target entrance and fall off regions.

When every detector module is equipped with a TDC, the scattered events can be suppressed in the offline analysis via the timing signature. The related reduction of statistics by less than $10 \%$ is tolerable.

\subsection{Influence of collimator configurations}

The results from the measurements with different collimator configurations are displayed in figure 15. The integrated signals (figure 11) of the three LYSO detectors behind the collimator slit are shown for each of the six different collimator configurations (table 2). Data have been taken at the target positions $-30 \mathrm{~mm}$ and $+40 \mathrm{~mm}$, respectively. The differences of the integrated count rates at these two positions are displayed for the six different collimator configurations in the upper part of figure 16. This contrast is more pronounced for the configurations with the collimator 'far' from the target (Nr. 0 to 3) as here the field-of-view is larger than at a 'close' configuration with the same slit width $s w$. For a better interpretation of the results from the three LYSO detectors the arithmetic mean is also shown in the figure (solid blue points). 

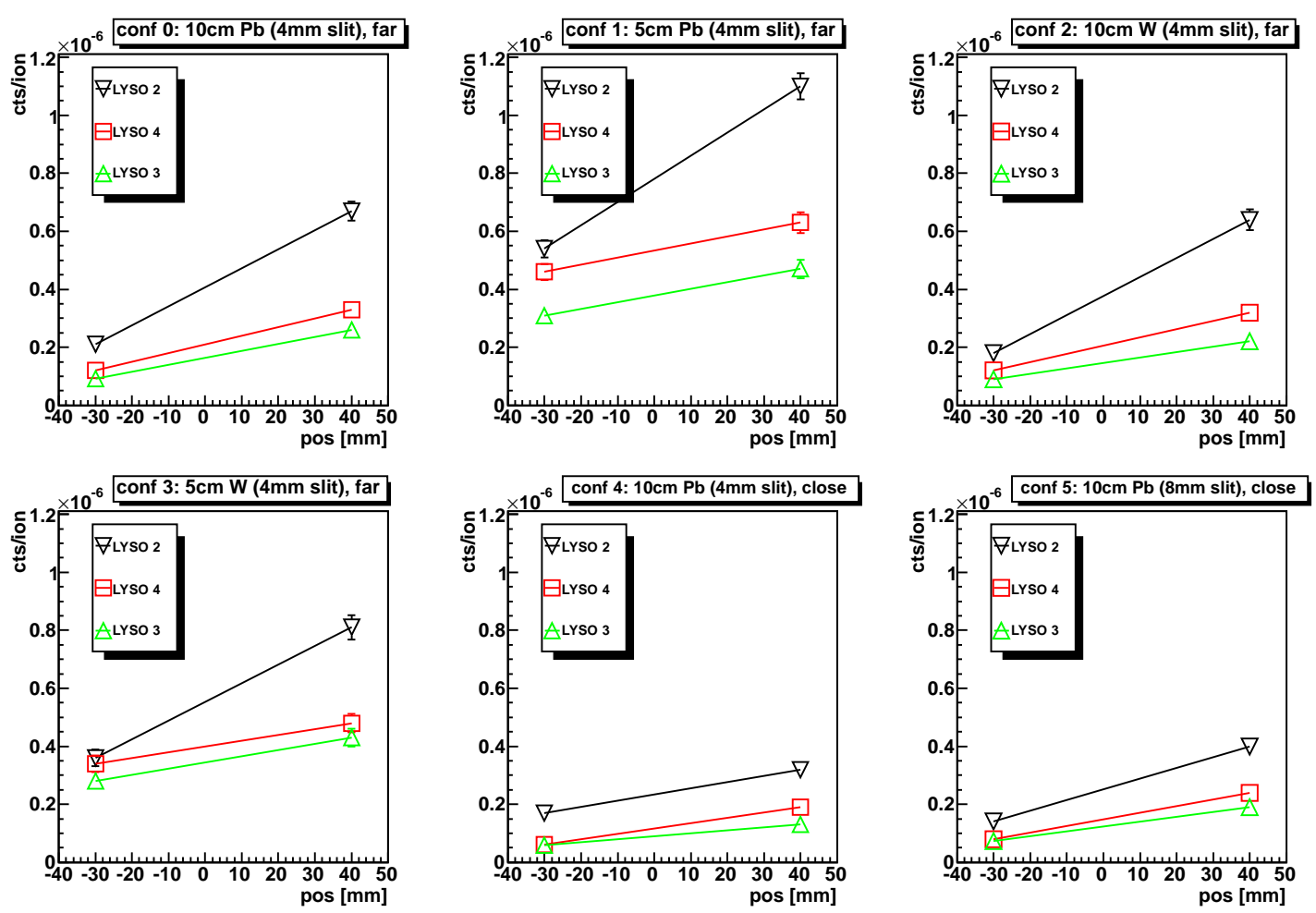

Figure 15. Integrated signals of the three LYSO detectors behind the collimator slit at the target positions $-30 \mathrm{~mm}$ and $+40 \mathrm{~mm}$ for the six different collimator configurations (table 2).

The lower part of figure 16 shows the contrast-to-noise ratio (CNR) where noise corresponds to the square root of the count rate at the point before the target $(-30 \mathrm{~mm})$. The CNR is reduced for the two configurations with thinner collimators ( $\mathrm{Nr} .1$ and 3 ) which do not provide complete absorption of the prompt gamma rays. The highest experimental CNR values are obtained with $100 \mathrm{~mm}$ thick collimators in the 'far' configuration (Nr. 0 and 2) followed by Nr. 5 where the collimator is closer to the target.

In this figure also the results from the Geant 4 simulations for the arithmetic mean CNR value of the three LYSO detectors are displayed (open circles). As mentioned above, more prompt gammas are produced and less background appears in the simulations, compared to experimental data. Nevertheless, even if absolute values cannot be reproduced, the results from Geant 4 nicely follow the trend from the experiment for the different configurations. For the CNR a factor 0.4 has been applied to the simulations for a better comparison with experiment. Here, a difference reveals between the two configurations with $100 \mathrm{~mm}$ collimators ( $\mathrm{Nr} .0$ and 2), which is not obvious from the experimental data alone. The simulation favors configuration Nr. 2 with the tungsten collimator. This result is in accordance with the shielding measurements described above.

\section{Conclusions}

As an intermediate step towards a clinical prototype of a collimated camera for online ion range monitoring during carbon-ion-therapy via the detection of prompt gamma rays, measurements have been performed with a multi-slit multi-detector configuration at a $75 \mathrm{MeV} / \mathrm{u}{ }^{13} \mathrm{C}$ beam impinging 

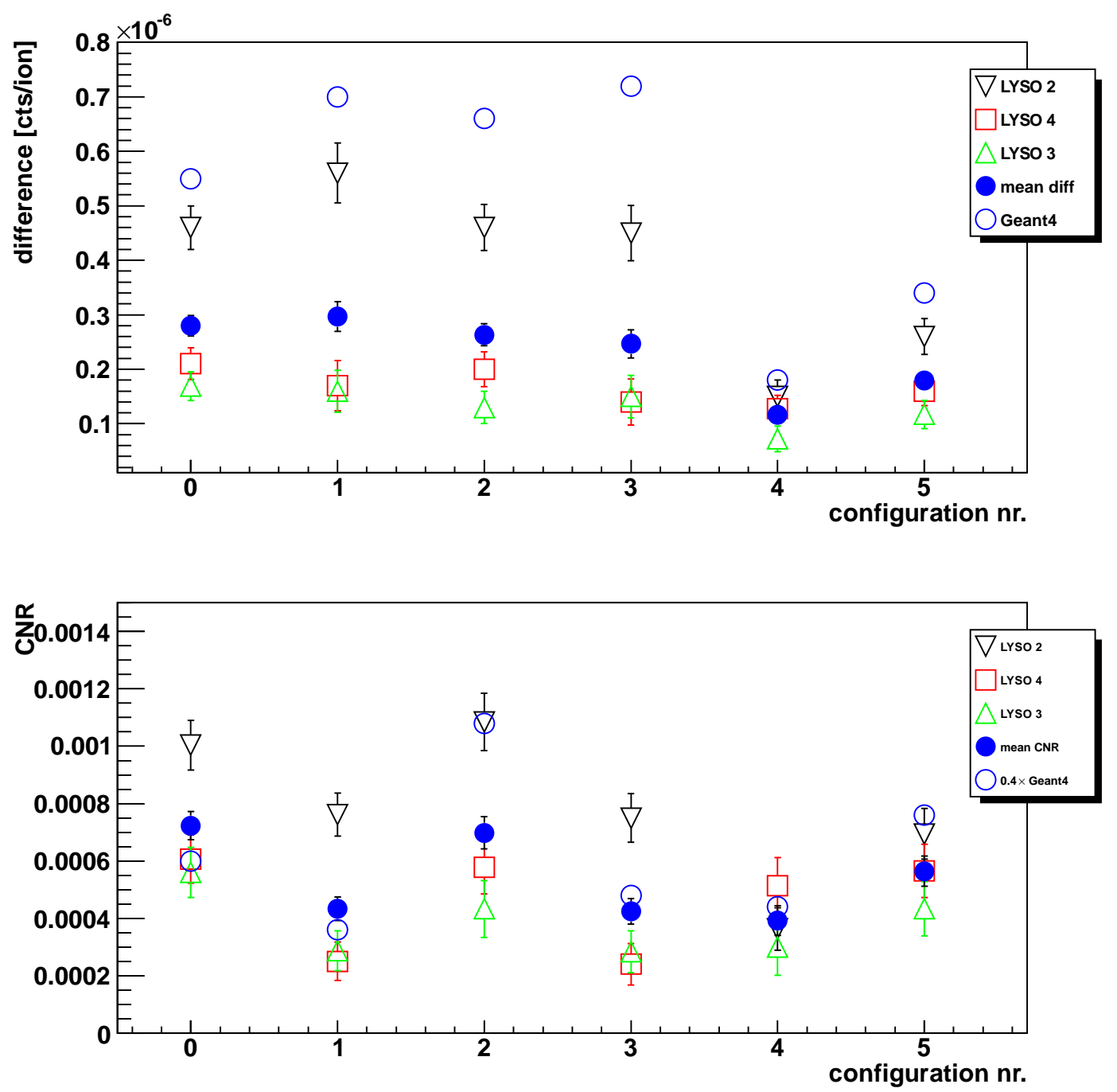

Figure 16. Up: difference of the integrated count rates at the two positions $(-30 \mathrm{~mm}$ and $+40 \mathrm{~mm})$ for the six different collimator configurations as a measure for the contrast. Down: contrast-to-noise ratio for the different configurations. For a better interpretation of the results the arithmetic mean from the three LYSO detectors has been calculated (solid blue points). Also shown are the results of the arithmetic mean from the simulations (open circles). For a better comparison with experiment, a factor 0.4 has been applied for the CNR (lower plot).

on a PMMA target. It has been shown that a selection of TOF and energy is necessary before information about the ion range can be extracted from the measurement of count rates at different target positions. The results for the five individual LYSO detector modules are in agreement with theoretical expectations. Prompt-gamma rays which undergo scattering in neighboring detector modules would dilute the information about the origin of the gamma rays and would therefore reduce the contrast. The fraction of these events has been determined in a separate experiment with $200 \mathrm{MeV} / \mathrm{u}{ }^{12} \mathrm{C}$ ions. From a combination of experimental results with simulations the portion of scattered events which are registered in a single detector module have been estimated to be on the 
$10 \%$-level. If necessary, these events can be discarded in the offline analysis, the related reduction in statistics is tolerable.

Different collimator configurations have been tested and shielding properties of lead and tungsten have been measured. The results from Geant 4 simulations are in agreement with experimental data, even if absolute prompt gamma production rates are overestimated by the simulations, the relative performance of the different collimator configurations could be reproduced. This confirms the use of Geant 4 for a further optimization of the collimator dimensions. Besides the signal contrast these simulations also take into account the range retrieval precision of the incident ions. The results from this collimator optimization will be subject of a forthcoming article [31].

\section{Acknowledgments}

This research project has been partly supported by the Regional Program for Research in Hadrontherapy (PRRH, under CPER 2007-13 funding), the European FP 7 projects ENVISION (grant agreement nr. 241851), ENTERVISION (grant agreement nr. 264552), ULICE (grant agreement nr. 228436) and the ANR Gamhadron project (ANR-09-BLAN-0106). This work was performed within the framework of the LABEX PRIMES (ANR-11-LABX-0063) and France Hadron (ANR11-INBS-0007).

\section{References}

[1] G. Shakirin et al., Implementation and workflow for PET monitoring of therapeutic ion irradiation: a comparison of in-beam, in-room, and off-line techniques, Phys. Med. Biol. 56 (2011) 1281.

[2] W. Enghardt et al., Charged hadron tumour therapy monitoring by means of PET, Nucl. Instrum. Meth. A 525 (2004) 284.

[3] T. Nishio, T. Ogino, K. Nomura and H. Uchida, Dose-volume delivery guided proton therapy using beam on-line PET system, Med. Phys. 33 (2006) 4190.

[4] U. Amaldi et al., Advanced quality assurance for CNAO, Nucl. Instrum. Meth. A 617 (2010) 248.

[5] P. Henriquet et al., Interaction vertex imaging (IVI) for carbon ion therapy monitoring: a feasibility study, Phys. Med. Biol. 57 (2012) 4655.

[6] C. Agodi et al., Charged particle's flux measurement from PMMA irradiated by 80 MeV/u carbon ion beam, Phys. Med. Biol. 57 (2012) 5667.

[7] K. Gwosch et al., Non-invasive monitoring of therapeutic carbon ion beams in a homogeneous phantom by tracking of secondary ions, Phys. Med. Biol. 58 (2013) 3755.

[8] C.-H. Min, C.H. Kim, M.-Y. Youn and J.-W. Kim, Prompt gamma measurements for locating the dose falloff region in the proton therapy, Appl. Phys. Lett. 89 (2006) 183517.

[9] C.H. Kim, C.H. Min, K.S. Seo and J.-W. Kim, Simulation studies on the correlation of distal dose falloff of a 70-MeV proton beam with a prompt gamma distribution, J. Korean Phys. Soc. 50 (2007) 1510 .

[10] E. Testa et al., Monitoring the Bragg peak location of $73 \mathrm{MeV/u}$ carbon ions by means of prompt gamma-ray measurements, Appl. Phys. Lett. 93 (2008) 093506.

[11] V. Bom, L. Joulaeizadeh and F. Beekman, Real-time prompt gamma monitoring in spot-scanning proton therapy using imaging through a knife-edge-shaped slit, Phys. Med. Biol. 57 (2012) 297. 
[12] J. Smeets et al., Prompt gamma imaging with a slit camera for real-time range control in proton therapy, Phys. Med. Biol. 57 (2012) 3371.

[13] M. Testa et al., Real-time monitoring of the Bragg-peak position in ion therapy by means of single photon detection, Rad. Env. Biophys. 49 (2010) 337.

[14] C.H. Min, H.R. Lee, C.H. Kim and S.B. Lee, Development of array-type prompt gamma measurement system for in vivo range verification in proton therapy, Med. Phys. 39 (2012) 2100.

[15] S.W. Peterson, D. Robertson and J. Polf, Optimizing a three-stage Compton camera for measuring prompt gamma rays emitted during proton radiotherapy, Phys. Med. Biol. 55 (2010) 6841.

[16] H. Seo et al., Experimental performance of double-scattering Compton camera with anthropomorphic phantom, 2011 JINST 6 C01024.

[17] M.-H. Richard et al., Design study of the absorber detector of a Compton camera for on-line control in ion beam therapy, IEEE Trans. Nucl. Sci. 59 (2012) 1850.

[18] S. Kurosawa et al., Prompt gamma detection for range verification in proton therapy, Curr. Appl. Phys. 12 (2012) 364.

[19] T. Kormoll, F. Fiedler, S. Schöne, J. Wüstemann, K. Zuber and W. Enghardt, A Compton imager for in-vivo dosimetry of proton beams — design study, Nucl. Instrum. Meth. A 626-627 (2011) 114.

[20] G. Llosá et al., First Compton telescope prototype based on continuous LaBr3-SiPM detectors, Nucl. Instrum. Meth. A 718 (2013) 130.

[21] F. Roellinghoff et al., Real-time proton beam range monitoring by means of prompt-gamma detection with a collimated camera, Phys. Med. Biol. 59 (2014) 1327.

[22] M. Pinto et al., Experimental carbon ion range verication in inhomogeneous phantoms using prompt gammas, submitted to Med. Phys. .

[23] R.B. Firestone, V.S. Shirley, C.M. Baglin, S. Chu and J. Zipkin, Table of Isotopes, John Wiley \& Sons Inc., New York U.S.A. (1996).

[24] K. Gunzert-Marx, H. Iwase, D. Schardt and R.S. Simon, Secondary beam fragments produced by $200 \mathrm{MeV} u^{-1}{ }^{12} \mathrm{C}$ ions in water and their dose contributions in carbon ion radiotherapy, New J. Phys. 10 (2008) 075003.

[25] J.M. Verburg, H.A. Shih and J. Seco, Simulation of prompt gamma-ray emission during proton radiotherapy, Phys. Med. Biol. 57 (2012) 5459.

[26] J.M. Verburg, K. Riley, T. Bortfeld and J. Seco, Energy- and time-resolved detection of prompt gamma-rays for proton range verification, Phys. Med. Biol. 58 (2013) L37.

[27] S. Agostinelli et al., Geant4-a simulation toolkit, Nucl. Instrum. Meth. A 506 (2003) 250.

[28] F. Le Foulher et al., Monte Carlo simulations of prompt-gamma emission during carbon ion irradiation, IEEE Trans. Nucl. Sci. 57 (2010) 2768.

[29] G. Dedes et al., Assessment and improvements of Geant4 hadronic models in the context of prompt-gamma hadrontherapy monitoring, Phys. Med. Biol. 59 (2014) 1747.

[30] J.H. Hubbell and S.M. Seltzer, Tables of $x$-ray mass attenuation coefficients and mass energy-absorption coefficients from $1 \mathrm{keV}$ to $20 \mathrm{MeV}$ for elements $z=1$ to 92 and 48 additional substances of dosimetric interest, http://www.nist.gov/pml/data/xraycoef/.

[31] M. Pinto et al., Design optimisation of a TOF-based collimated camera prototype for online hadrontherapy monitoring, Phys. Med. Biol. 59 (2014) 7653. 